\title{
Plume Particle Energy Analysis of an Ionic Liquid Electrospray Ion Source with High Emission Density
}

\author{
C. $\mathrm{Ma}^{1, \mathrm{a})}$ and C. Ryan ${ }^{1, \mathrm{~b})}$ \\ Faculty of Engineering and Physical Sciences, University of Southampton.
}

(Dated: 28 December 2020)

A retarding potential analyser was used to characterise the energy distribution of the plume particles from an electrospray source. The electrospray device uses an ionic liquid, operates at bipolar and relatively high voltages from \pm 1800 to $\pm 3500 \mathrm{~V}$, and demonstrated ionic emissions with relatively high emission density of more than $\pm 30 \mu \mathrm{A}$ per emission tip. Electrostatic simulations were used to study the effects of electric field distortion near the grids in the retarding potential analyser, and a correction factor of $93 \%$ was used to regulate the deceleration voltage in the energy analysis, from which the voltage losses between the applied voltage of the electrospray source and the actual acceleration voltage of the charged particles were calculated, demonstrating non-kinetic efficiency from $85.8 \%$ at $-2100 \mathrm{~V}$ to $79.6 \%$ at $2600 \mathrm{~V}$. The plume particle energy analysis shows evidence of fragmentation of heavier particles, mostly from dimer ions to monomer ions, and detailed energy analysis was used to estimate the position where the fragmentation occurs. The results suggest that about $45 \%$ to $55 \%$ of the particle fragmentation occurred in the field-free region, $20 \%$ to $30 \%$ occurred in the acceleration region with an intense electric field, with the rest of the plume containing unfragmented ions.

\section{INTRODUCTION}

In an electrospray source, an electric field is applied between a liquid and a downstream electrode to extract and accelerate charged particles from the liquid surface. The liquid is usually transported from a reservoir to a protruding structure, termed an emitter, and forms a Taylor cone on the emitter tip as a result of the equilibrium condition between the liquid surface tension and surface electrical stress. The emitted particles are usually droplets, and in some examples, ions. The main applications of electrospray are in mass spectrometry $^{1-3}$, and other uses have been investigated and developed, including electrospinning fabrication ${ }^{4,5}$, ion beam etching $^{6-8}$, ion beam deposition ${ }^{9,10}$ and space propulsion for micro/nano-satellites ${ }^{11-14}$.

The electrospray ion source discussed in this paper, termed Porous-Emitter Ion Liquid Ion Source (PEILIS), was initially developed at the University of Southampton for the purpose of a space propulsion system, the Porous-emitter Electrospray Thruster with 100 emission tips (PET-100). The PEILIS electrospray ion source features a compact design and uses an ionic liquid, 1-Ethyl-3-methylimidazolium tetrafluoroborate $\left(\mathrm{EMI}_{-} \mathrm{BF}_{4}\right)$, as the electrospray medium. The PEILIS has a emitter made of porous borosilicate glass, which has 100 pyramidal-shaped electrospray emission tips that were fabricated using computer-numerical-controlled (CNC) machining. The liquid is transported to the porous emitter from a porous reservoir solely relying on capillary action, as illustrated in Fig. 1. The PEILIS device was tested in a vacuum in a bipolar electrical mode. With the extractor kept at the common ground, the potential of the propellant is periodically switched between positive and negative potential, extracting anions and cations from the liquid surface to eliminate charge accumulation. The emission current was found

\footnotetext{
a)Electronic mail: c.ma@soton.ac.uk

${ }^{b)}$ Electronic mail: c.n.ryan@soton.ac.uk.
}

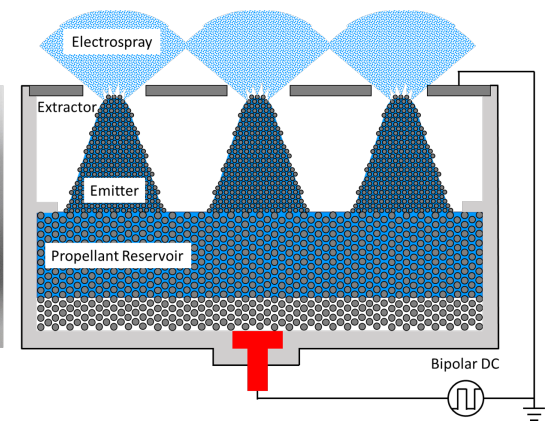

FIG. 1. A porous-emitter ion liquid ion source (PEILIS).

generally stable and its voltage-current characteristics were measured. Compared to other electrospray ion sources using porous emitters, PEILIS demonstrated noticeably high overall electrospray emission current, as well as the emission current density per emission tip. The plume particle composition was characterised using a time-of-flight system, and the results suggested the plume contain mostly monomer ions, $\mathrm{EMI}^{+}$and $\mathrm{BF}_{4}{ }^{-}$, and dimer ions, $\left(\mathrm{EMI}-\mathrm{BF}_{4}\right) \mathrm{EMI}^{+}$and (EMI$\left.\mathrm{BF}_{4}\right) \mathrm{BF}_{4}{ }^{-}$, with no evidence of droplets presence. With the applied voltages up to $\pm 3500 \mathrm{~V}$, the maximum overall emission currents achieved by PEILIS device were $+3.22 \mathrm{~mA}$ and -4.72 $\mathrm{mA}( \pm 30 \sim 40 \mu \mathrm{A} \text { per emission tip })^{13}$, respectively, significantly higher than those from other electrospray models using a similar design architecture (generally less than $1.5 \mu \mathrm{A}$ per emission tip) $)^{11,14}$. Apart from the high emission output, the energy of the ions emitted from the PEILIS device was relatively high, ranging from 2.5 to $3.5 \mathrm{keV}$, with ion velocities generally higher than $40 \mathrm{~km} / \mathrm{s}$.

With dimer ions contained in the emission using the EMI$\mathrm{BF}_{4}$ electrospray medium, fragmentation of the dimer ions into a monomer ion and a neutral molecule is likely to occur, an effect found in multiple other studies ${ }^{14-16}$. In this study, the energy distribution of the PEILIS plume particles was measured using a retarding potential analyser (RPA) to 
characterise the fragmentation and the corresponding effects on the electrospray performance, including the mass, velocity and energy uniformity of the emitted species. This paper introduces a retarding potential analysis system designed to characterise a PEILIS electrospray source, and discusses the measurement results along with the implications on the electrospray ionic liquid ion source performance.

\section{A RETARDING POTENTIAL ANALYSIS SYSTEM}

\section{A. RPA Design}

An RPA typically consists of a plume particle collector and three upstream grids applied with different electric potentials. The most upstream grid is kept at ground potential. As a crucial component, the second grid in the downstream (i.e. the RPA-HV grid) is applied with a variable potential sweeping from ground to a potential higher than that of the emitter. As the applied potential on the RPA-HV grid increases, the particles that have entered the RPA will be electrostatically decelerated until stopped before this grid. As a result, the collected plume current decreases with the applied potential on the RPA-HV grid, from which the energy distribution of the charged plume particles can be calculated. The other grid is applied with a negative potential used to suppress the emission of secondary electrons, which can be placed between the ground grid and the RPA-HV grid $^{17}$ or between the RPA-HV grid and the RPA collector ${ }^{11,16}$.

In this work, the RPA probe featured a four-grid design with two secondary-electron-suppression grids, as illustrated in Fig. 2. The front-panel aperture is $30 \mathrm{~mm}$ in diameter. The nickel grids had $90 \%$ transparency with an opening width of $344 \mu \mathrm{m}$ and wire line width of $19 \mu \mathrm{m}$, sourced from Precision Eforming LLC. With four grids, the overall transparency is $65.61 \%$, which was used to proportionally correct the particle current calculations. This study takes the assumption that different particle species in the plume would experience similar collision behaviours in the electrostatic system, and as a result, the grid transparency has little to no influence on the measurement results of particle energy distribution. The gap between adjacent grids is $2 \mathrm{~mm}$, and the collector is placed 5 $\mathrm{mm}$ in the downstream of the fourth grid. The RPA was tested inside a vacuum chamber, and the grid potentials were supplied from power sources outside the chamber via threaded bolt connectors.

\section{B. Electrostatic Field Simulation}

This study defines a critical equipotential surface within the RPA, which has the required relative-to-ground potential that can electrostatically stop all the high-energy ions flying toward the RPA collector. As a critical factor determining the effectiveness of the electrostatic deceleration, the critical equipotential surface must cover the entire cross-section area of the aperture in order to repel all the ions of given a energy. However, the electric field generated between adjacent grids

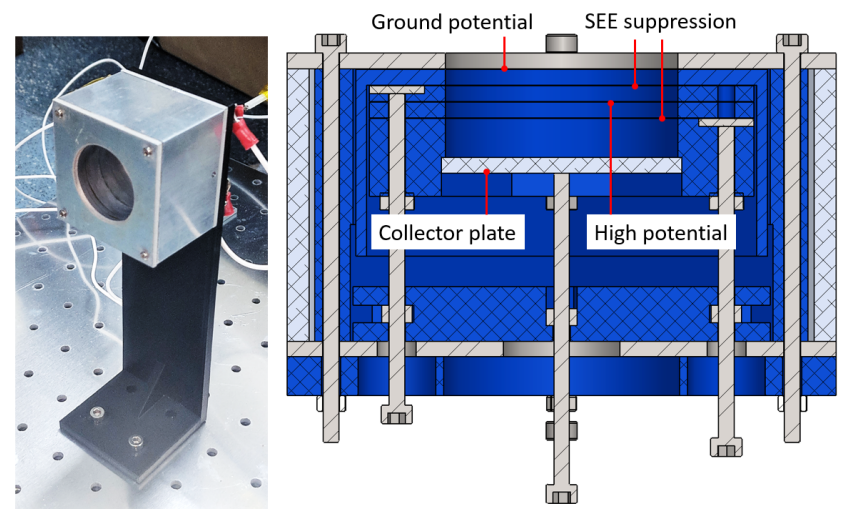

FIG. 2. Design of the retarding potential analyser.

applied with different potentials would cause interference to the electric field close to the grid aperture; therefore, the geometries of the equipotential surfaces are always curved or even self-enclosed near the grid edges. Such effects are more substantial with closer distances between adjacent grid layers with significant potential differences. As a result, the equipotential surface covering the entire aperture is always lower than the potential applied to the grid, and consequently, the RPA measurement results will need to be calibrated based on such differences.

In order to quantitatively analyse the differences between the applied grid potential and the equipotential that covers the entire aperture, an electrostatic simulation model considering charged particle tracing was built using COMSOL Multiphysics software, version 5.4. The model geometry has three grid layers and a collector layer, whose distances are the same as in the design. As each grid contains thousands of openings, in order to reduce the computational cost, only one grid opening is considered in these simulations. The side boundaries of the cuboid computation domain are set to have periodical conditions, which represent an infinitely broad grid that considers electric field interference from adjacent openings. In order to simulate the trajectory of charged particles, a region near the top surface of the long cuboid computation domain is set as an acceleration region, where an acceleration voltage is applied along the long axis. A group of ions randomly distributed on the top surface enter this region given zero initial velocity and are electrostatically accelerated before leaving the acceleration region, representing the high-energy particles emitted from the electrospray source.

The emission rates of ions to be studied in the model can be estimated from initial current collected by RPA with floating RPA-HV grid potential. The emission current intensities at different voltages

Assuming all particles in the RPA are singly charged and each grid in the RPA has the same transparency in intercepting ions, the formula of the quantitative rates of ions passing through the RPA aperture, $r_{i}$, is

$$
r_{i}=\frac{I_{r p a}}{t^{n} e}
$$

where $I_{r p a}$ is the partial plume current received on the RPA 
TABLE I. Initial RPA collector current values at different emitterextractor voltages with the RPA-HV grid potential of $0 \mathrm{~V}$.

\begin{tabular}{lcr}
\hline \hline$V_{\text {applied }}(\mathrm{V})$ & $I_{\text {rpa }}(\mu \mathrm{A})$ & $I_{r p a}$ error $(\mu \mathrm{A})$ \\
\hline-2100 & -0.055879 & \pm 0.0228 \\
-2200 & -0.081826 & \pm 0.0241 \\
-2400 & -0.26014 & \pm 0.0330 \\
2100 & 0.0485 & \pm 0.0224 \\
2200 & 0.0765 & \pm 0.0238 \\
2300 & 0.1522 & \pm 0.0276 \\
2400 & 0.276 & \pm 0.0338 \\
2500 & 0.31 & \pm 0.0355 \\
2600 & 0.38 & \pm 0.0390 \\
3000 & 0.75 & \pm 0.0575 \\
\hline \hline
\end{tabular}

collector, $e$ is the electron charge, $t$ is the transparency of one grid and $n=4$ is the number of grids in the RPA system. The RPA tests were conducted with different voltages between the emitter and the extractor, and some of the corresponding initial RPA collector current values are shown in Table I. The error ranges estimated were based on three sources: signal noises in the data acquisition system, the millisecond-level delay between giving control command and actuating voltage output from the power supply, and the inherent, despite reasonably minor, instability of the PEILIS electrospray emission.

The highest RPA current was $0.75 \mu \mathrm{A}$ at $3000 \mathrm{~V}$, corresponding to the ion emission rate of approximately 7134.8 per nanosecond. Comparing the area of each opening to the overall RPA aperture area, and assuming the ions are evenly distributed across each grid opening in a random manner, the ion passing rate per grid opening is approximately 1.33 per nanosecond. On this basis, in these simulations, the particle releasing rate is set as one per nanosecond from a pseudorandom position on the emitter plane. In order to simplify the simulation, all the ions are assumed to be dimer ions without any monomer ions. The solutions are calculated every $0.2 \mathrm{~ns}$ over a total duration of $500 \mathrm{~ns}$, which is long enough for the first-released ions to reach the RPA collector plane. Coulomb forces between particles are considered. These particles fly 5 $\mathrm{mm}$ distance before reaching the first ground grid of the RPA system. For example, with an acceleration voltage of $3000 \mathrm{~V}$, an RPA-HV grid potential of $3000 \mathrm{~V}$ and an RPA-SEE grids potential of $-30 \mathrm{~V}$, a cross-section view of the overall electric field is shown in Fig. 3, which was calculated using electrostatic Laplace's equation without considering space charge effects induced by the ions.

The results suggest that a RPA-HV grid applied with 3000 $\mathrm{V}$ potential cannot stop the particles from reaching the collector, and a higher RPA-HV grid potential is required to stop all the particles from passing through the grid aperture. In order to quantitatively analyse the potential required, another particle trajectory simulation case was studied with the RPA-HV grid potential of $4000 \mathrm{~V}$. With the RPA-HV grid potential of $3000 \mathrm{~V}$ and $4000 \mathrm{~V}$, some equipotential surfaces close to the grid are shown in Fig. 4, where the spatial surface plots use the same electric potential colour legend and the particle trajectories use the same velocity magnitude colour legend. It



FIG. 3. The overall simulation geometry and electric field simulation results.

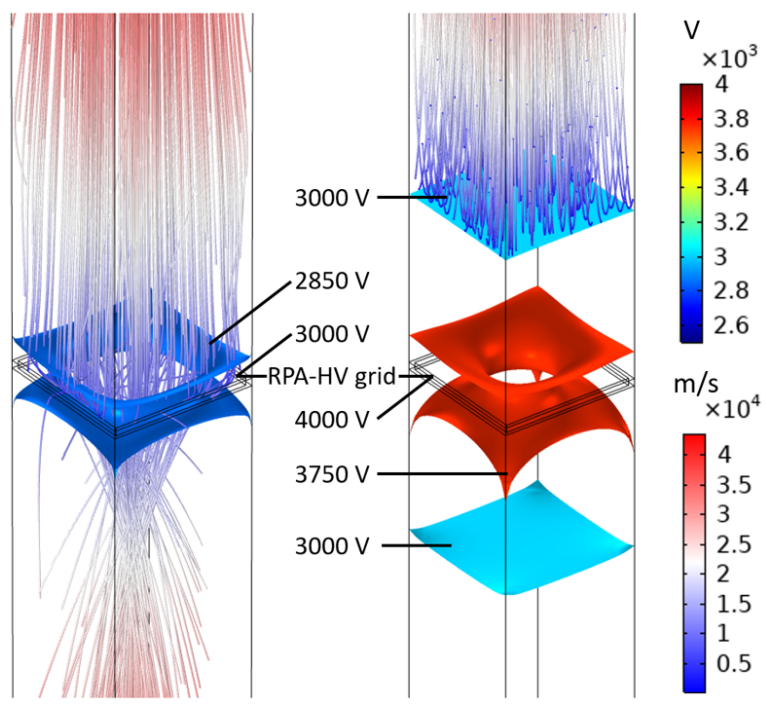

FIG. 4. 3D line plots are particle trajectory simulation results with the RPA-HV grid potential of $3000 \mathrm{~V}$ (left) and $4000 \mathrm{~V}$ (right), and their colour legend shows the velocity magnitude. Several equipotential surfaces are plotted, demonstrating the distorted electric field near the grid and their effects on the energy of ions, whose incoming energy is $3000 \mathrm{eV}$ per particle.

can be found that all of monomer ions with $3000 \mathrm{eV}$ energy are stopped before reaching the aperture of the RPA-HV grid with $4000 \mathrm{~V}$ potential.

The electric potential spatial surface plots in Fig. 4 clearly show hollowed geometries at the central area, where the electric potentials are less than the grid potential. The twodimensional equipotential lines on the central cut-plane near the RPA-HV grid are shown in Fig. 5. The minimum potentials covering the entire aperture, shown as the curved $\mathrm{X}$ shaped equipotential lines at the center, are $2783 \mathrm{~V}$ and 3724 $\mathrm{V}$ in the two scenarios with the RPA-HV potentials of $3000 \mathrm{~V}$ and $4000 \mathrm{~V}$, respectively.

In these RPA simulation, the RPA-HV grid potential is only 


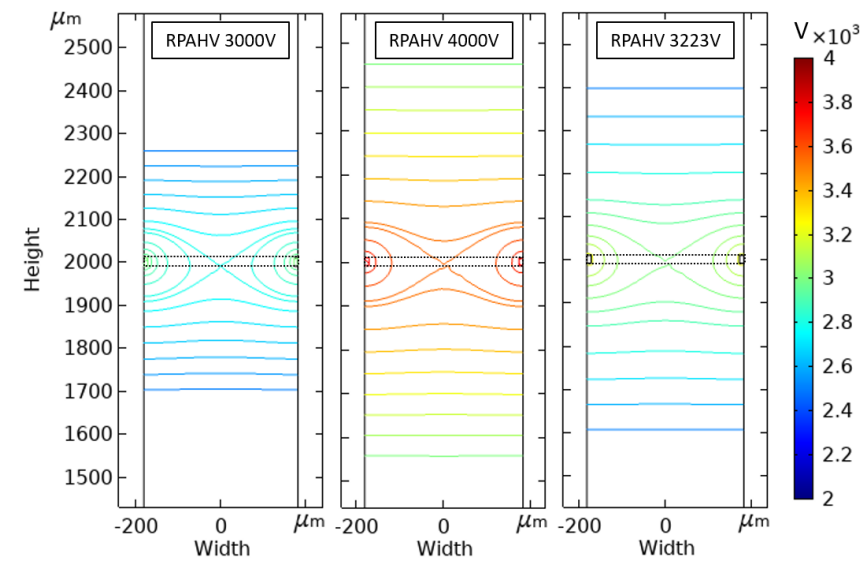

FIG. 5. 2D equipotential lines with different RPA-HV grid potentials. The RPA-HV grid is located at the $2 \mathrm{~mm}$ height. The three subfigures share the same colour legend of electric potential.

one variable, and thus, a voltage ratio, $k$, can be defined as

$$
k=\frac{U_{c}-U_{\text {see }}}{U_{h v}-U_{\text {see }}}
$$

where $U_{c}$ is the minimum potential value of the equipotential spatial surfaces covering the entire grid aperture, $U_{h v}$ is the potential of the RPA-HV grid, and $U_{\text {see }}$ is the potential of the RPA-SEE grid. For simulations with different RPA-HV potentials, the values of $k$ are similar, calculated as approximately 0.93 in these two simulation cases. In order to find the minimum RPA-HV grid potential that has a $3000 \mathrm{~V}$ equipotential surface covering the entire aperture, the $U_{c}$ is assigned as $3000 \mathrm{~V}$, and the minimum value of $U_{h v}$, required to stop all the incoming particles, is calculated as approximately $3223 \mathrm{~V}$. The simulation results with the $3223 \mathrm{~V}$ RPA-HV grid potential are also shown in Fig. 5, where the central curved X-shaped equipotential line is $3000 \mathrm{~V}$, proving this calculation method is indeed valid. When analysing the RPA measurement results that are discussed in the following sections, the $k$ factor was used to correct the actual stopping potential from the applied RPA-HV grid potential.

\section{Test Apparatus}

The measurements were conducted using the vacuum system in the David Fearn Electric Propulsion Laboratory, with the vacuum chamber size of two metres in diameter and four metres in length. The vacuum system is fitted with one roughing pump, two turbo pumps and one cryogenic panel, capable of maintaining a high vacuum with a background pressure around $9.8 \times 10^{-6}$ mbar during the PEILIS electrospray testing. The PEILIS electrospray device was mounted on a rotational platform. The RPA was mounted on the plate, with its collector surface approximately $35 \mathrm{~cm}$ away from the PEILIS extractor. In order to capture the overall plume current, a 15 $\times 15 \mathrm{~cm}$ current collector was placed $5 \mathrm{~cm}$ from the extractor at a perpendicular direction.

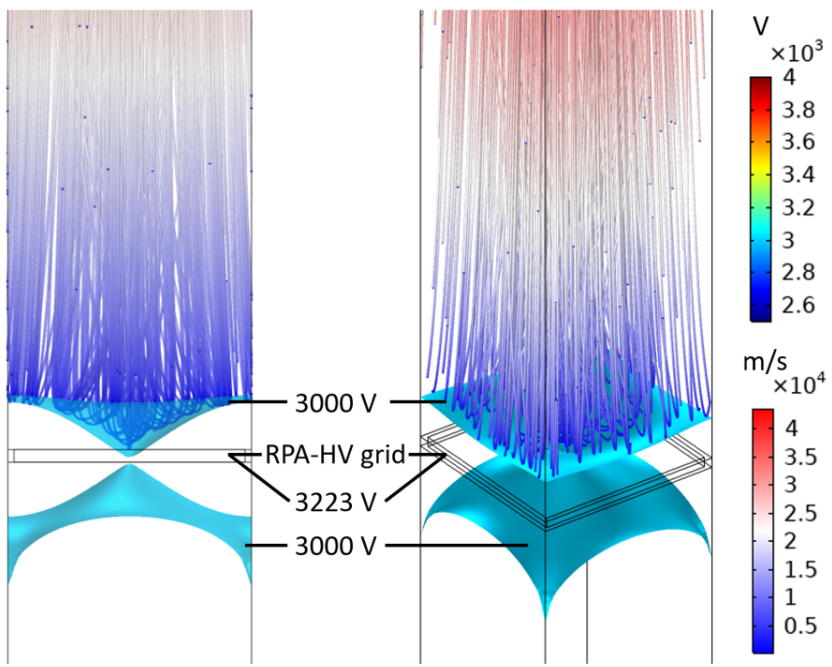

FIG. 6. Simulation results of electrostatic field and particle trajectories with the RPA-HV potential of $3223 \mathrm{~V}$. All the ions are stopped at the $3000 \mathrm{~V}$ equipotential surface near the center of the RPA-HV grid.

The electrical set up of the RPA measurement system is illustrated in Fig. 7. The electric potential of the two SEE suppression grids were kept at $-30 \mathrm{~V}$ during the tests, supplied by a DC power source outside the vacuum chamber. The potential of the RPA-HV grid is applied using a non-programmable HCP 350-12500 high voltage power supply. The power supply knob was integrated with an Arduino controlled stepper motor to control the voltage output stable. The rising time of the RPA sweep voltage was limited by the rotational speed and accuracy of the stepper motor. In order to maintain a high signal fidelity with reasonable synchronisation accuracy, the emitter electrical polarity of the PEILIS was switched every $30 \mathrm{~s}$, allowing a full-range measurement of RPA voltages, from 0 to $\pm 3500 \mathrm{~V}$, to be completed within one emission polarity. The collected RPA current data is transferred to a 4262 PicoScope through a FEMTO DHPCA-100 current amplifier with $1 \mathrm{MHz}$ bandwidth limit.

\section{MEASUREMENT RESULTS}

As a typical example, an RPA current data curve collected at $+2500 \mathrm{~V}$ emitter voltage is shown in Fig. 8(a). There were mild high-frequency noises contained in these signals, which were filtered using a low-pass Butterworth filter. As the RPA sweep voltage increases to the applied electrospray voltage, the collected current gradually decreases until reaching nearzero values. The variation of the collected current can be divided into five parts, corresponding to different physical effects occurring, as shown in Fig. 8(a).

During Stage 1, the collected current maintains a similar value as no charged particles are retarded, until it enters Stage 2 at approximately $800 \mathrm{~V}$, where the current drastically decreases by approximately $65 \%$ with a minor voltage increase 


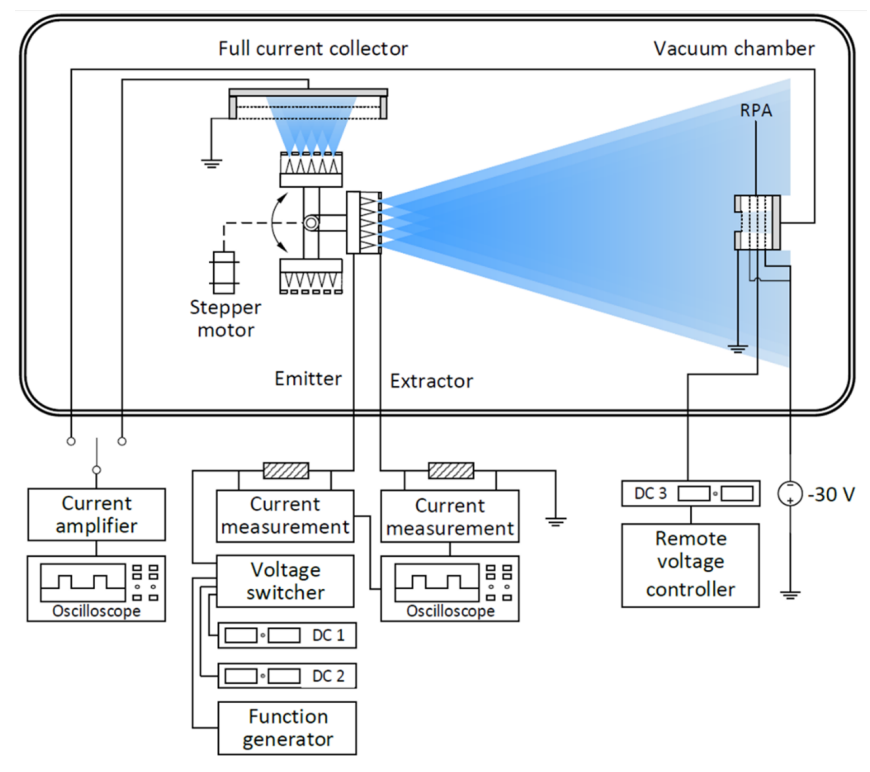

FIG. 7. Retarding potential analysis system and electrical setup.

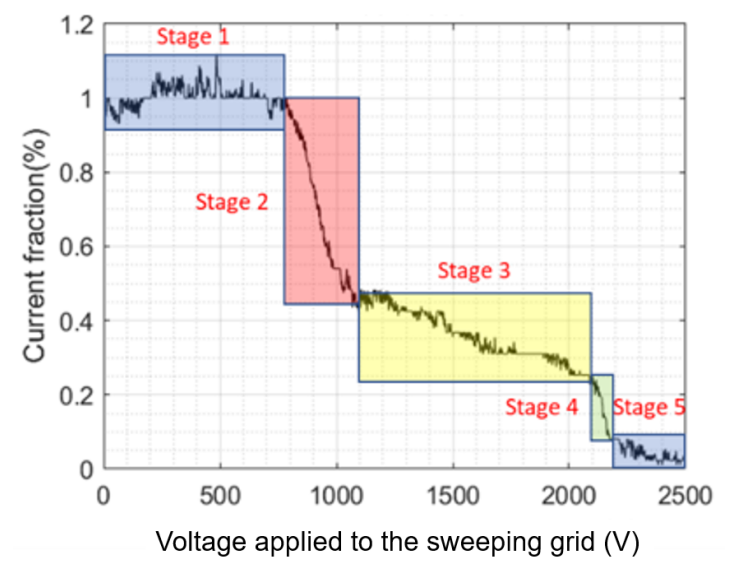

(a)Current measurement data.

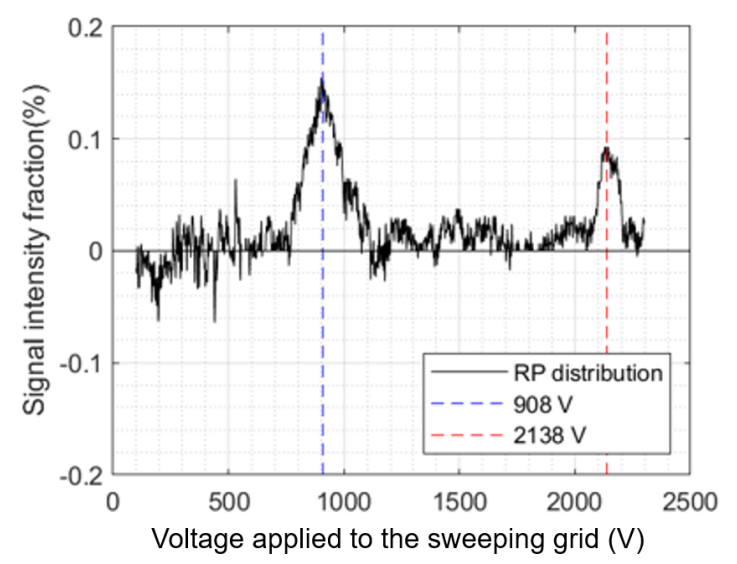

(b)Current intensity with 200 steps interval.

FIG. 8. RPA measurement results at $+2500 \mathrm{~V}$. by approximately 300 V. During Stage 3, the collected current gradually decreases at a moderate rate. Stage 4 involves another significant decrease in current by approximately $18 \%$, followed by a relatively constant near-zero value in Stage 5.

Stage 2 and Stage 4, with the most significant current decreases, indicate the energy range and the quantitative proportion of the majority of the particles in the plume. Stage 3, where the current gradually decreases, suggests a small fraction of the charged particles have a relatively wide energy distribution. The overall current data curves share similar trends with some other RPA studies of electrospray sources ${ }^{14,17,18}$, where the five steps have also been found.

The differentiated RPA data can highlight the signal intensity of particles with different energies. However, the current data has high-frequency noises, which creates misleading differentiated values if the calculations are based on any adjacent two points. Instead, the differentiated values were calculated based on two data points with a certain number of interval steps. A short interval, such as 10 , would not yield meaningful data for differentiation analysis, while an overly broad interval, such as 400, would reduce the fidelity of the differentiated data. Using intervals within a moderate range would result in a smooth current intensity curve while keeping a reasonably high data fidelity, with an example shown in Fig. 8(b) that has an interval length of 200 data points.

These methods of data post-processing were also performed on other RPA measurement data at different voltages, with the Gaussian-filtered results shown in Fig. 9 and Fig. 10. All the collected RPA current data curves indicate the five different stages, but the RPA-HV grid potential that has the highest signal intensity increases with the applied emitter voltage. It should be noted that all the retarding potentials shown in these figures have been corrected using the $k$ factor as discussed in the previous simulation section.

\section{ENERGY LOSS AND NON-KINETIC EFFICIENCY}

The non-kinetic power efficiency takes into account the voltage loss through the resistors in the circuit, the propellant resistance and the necessary voltage for extracting ions from the liquid propellant ${ }^{19}$. It is the ratio of the actual acceleration voltage compared to the input voltage that can be expressed as

$$
\eta_{n k}=\frac{V_{a c}}{V_{i n}}=\frac{V_{i n}-V_{e x}-R_{e m} I_{e m}}{V_{i n}},
$$

where $V_{\text {in }}$ is the input voltage between the power connection point of the emitter and the extractor, $V_{\text {loss }}$ is the overall voltage loss in the system consisting of $V_{e x}$ and $R_{e m} I_{e m}, V_{e x}$ is the extraction voltage of the emitted particles and $R_{e m}$ and $I_{e m}$ are the electrical resistance and current flow of the emitter.

In this RPA study, the voltages of current intensity peaks in Stage 4 are slightly smaller than the applied emitter voltage (i.e. the current decays to zero at an RPA voltage lower than the emitter voltage), and they represent the energy of the charged particles without fragmentation. These charged particles were considered fully energized by the electric acceleration field; therefore, their energy can be used to calcu- 


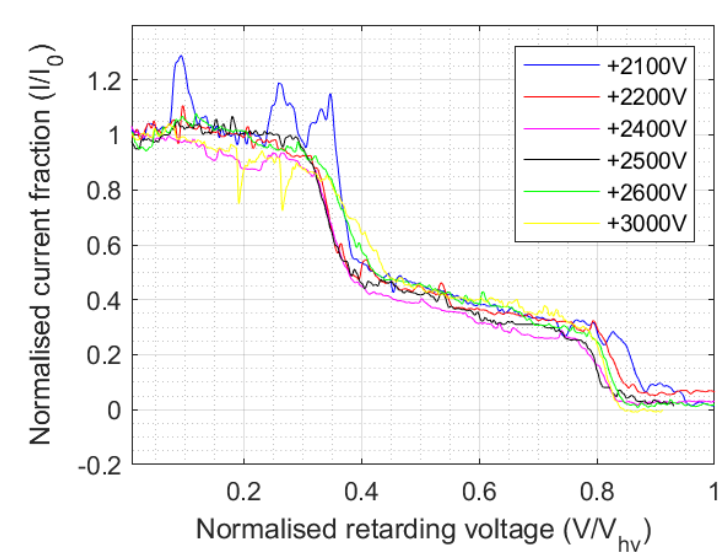

(a)Positive voltages.

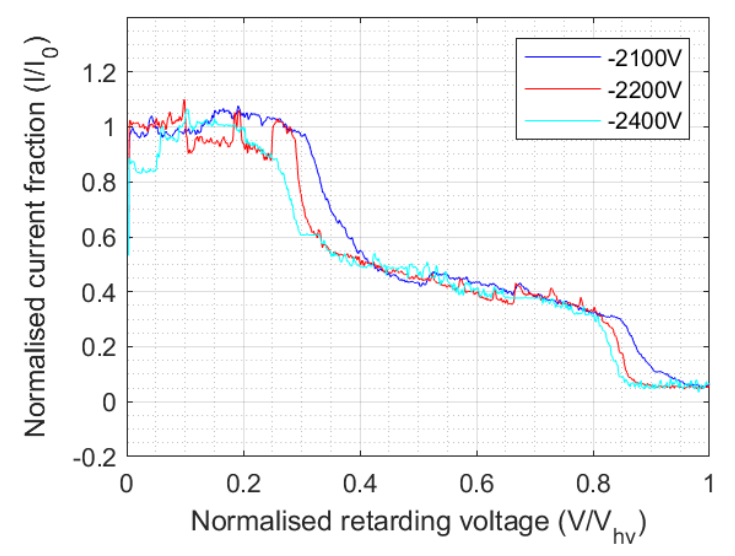

(b)Negative voltages.

FIG. 9. Normalised RPA current measurement results at different voltages.

late the value of acceleration voltages as well as the overall voltage losses, which are the differences between the acceleration voltages and the applied emitter voltages. The loss of voltage possibly resulted from the conducting voltage loss and the extraction voltage loss, from which the non-kinetic energy efficiency can be calculated, as listed in Table II and shown in Fig. 11. The actual acceleration voltage data points are selected as the second major current intensity peak points shown in Fig. 10, in a similar manner to the selection of the peak points shown in Fig. 8(b). Similar phenomena to the spread of current drops in Stage 4 were found in some other studies ${ }^{14,16-18,20}$, and a possible reason is that the plume particles are indeed not mono-energetic, or it could relate to the properties of the RPA measurement device used. The error ranges in the voltage losses are taken as the spread widths of Stage 4 current drops, which are of approximate values as the current intensity peak widths.

The results show that the loss of voltage increases with the voltage applied to the emitter. Through comparing the voltage loss data with volt-ampere curves collected using the large collector plate with a SEE suppression voltage of -100 $\mathrm{V}$, it can be found that the trends of these two data are gen-

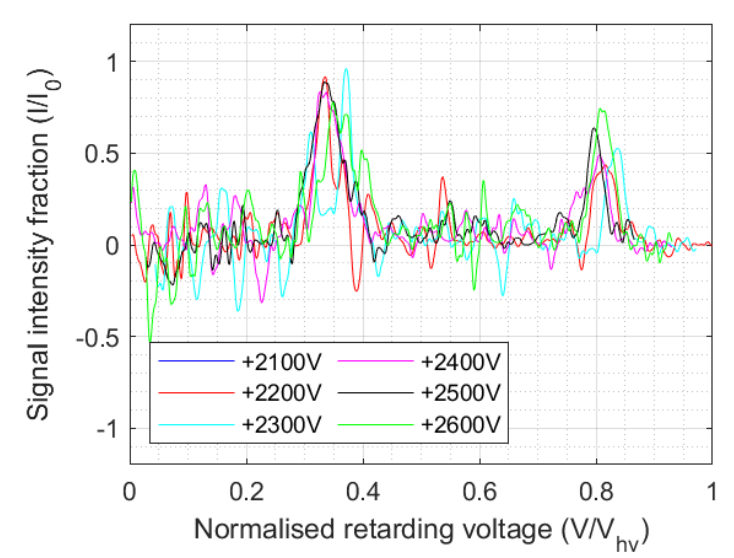

(a)Positive voltages.

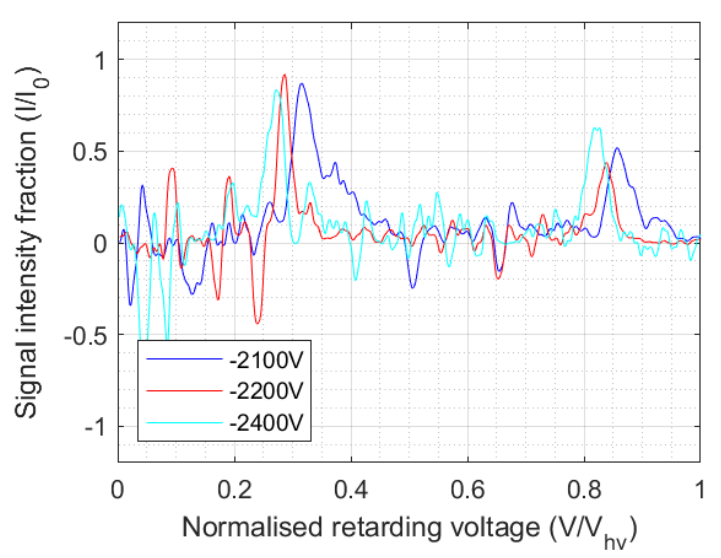

(b)Negative voltages.

FIG. 10. Normalised RPA current intensity results at different voltages.

TABLE II. Voltage loss and non-kinetic energy efficiency at different voltages.

\begin{tabular}{lcccc}
\hline \hline$V_{\text {applied }}(\mathrm{V})$ & $\eta_{n k}(\%)$ & $\eta_{n k}$ error $(\%)$ & $V_{\text {loss }}(\mathrm{V})$ & $V_{\text {loss }}$ error $(\mathrm{V})$ \\
\hline-2100 & 85.8 & -2.10 to +4.55 & 298 & -44.0 to +95.5 \\
-2200 & 84.1 & -3.78 to +2.56 & 350 & -83.1 to +56.4 \\
-2400 & 82.5 & -1.89 to +3.15 & 420 & -45.3 to +75.6 \\
2100 & 84.9 & -2.96 to +1.46 & 317 & -62.2 to +30.8 \\
2200 & 81.8 & -2.32 to +4.44 & 401 & -51.1 to +97.7 \\
2300 & 83.4 & -1.34 to +2.30 & 381 & -30.8 to +52.9 \\
2400 & 80.5 & -3.76 to +2.05 & 468 & -90.3 to +49.2 \\
2500 & 79.6 & -1.46 to +3.38 & 511 & -36.5 to +84.4 \\
2600 & 80.7 & -1.98 to +3.74 & 503 & -51.5 to +97.3 \\
\hline \hline
\end{tabular}

erally well matched, as shown in Fig. 11. Theoretically, the extraction voltage is a result of the electrical stress required to exceed the monomer or dimer ionic bond strength, and it is envisaged to have a constant value independent of the applied emitter-extractor voltage. Fig. 11 illustrates though that the total voltage loss increases with the applied voltage and the emission current. In comparison, assuming the EMI$\mathrm{BF}_{4}$ liquid conductivity does not vary during the electrospray 


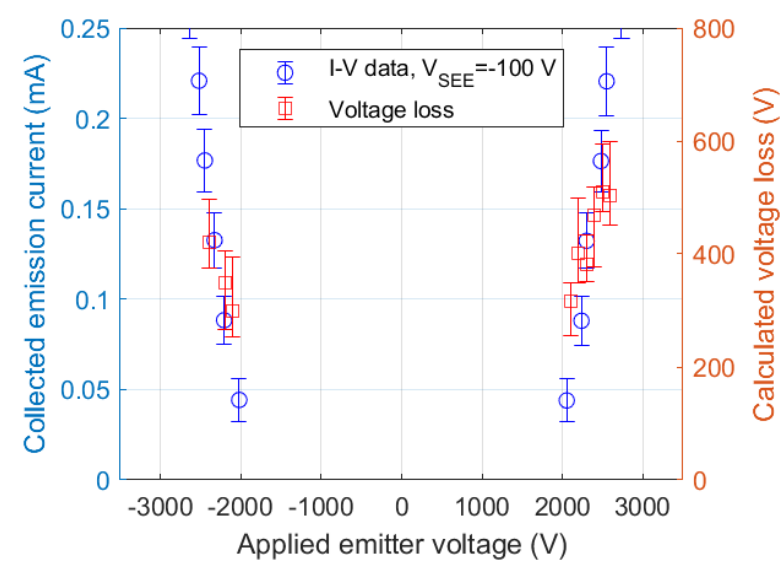

FIG. 11. Comparison of voltage losses calculated from RPA measurement results and the current-voltage curve of the electrospray source.

process at different voltages, the voltage loss caused by the propellant resistance would increase with the emitter current, which agrees with the measurement results. Therefore, it can be concluded that the resistance of propellant liquid is likely the main cause of voltage losses at higher emitter voltages. These results suggest that the effects of propellant liquid resistance on voltage loss are not negligible, providing feedback to the design of the PEILIS electrospray device on the emitter material selection. For example, the voltage loss might be greatly dependent on the details of the emitter design, the relatively large emitters used in this study (2-mm-tall tips with a 3-mm-thick substrate) may be conceivably liable to the result in higher electrical resistance than smaller emitters. This study only investigated the overall voltage losses with different emitter-extractor voltages, but it cannot identify the proportion of voltage loss from different sources, which will require further investigation.

\section{FRAGMENTATION EFFECTS}

Ideally, the emitted particles of an electrospray source are mono-energetic with a value close to the applied voltage, similar to Stage 4 in Fig. 8(a). The five-stage energy distribution can be a result of several possible reasons. One possibility is that the emitted particles contain different species including ions and droplets, with the different species having significantly different extraction voltages, resulting the two main current drops as illustrated in the RPA measurement data. However, this is unlikely to be true as these two stages have rather concentrated energy distribution, while electrospray droplets normally have a wider mass distribution and hence would exhibit a wider energy distribution. Also, previous time-of-flight characterisations regarding PEILIS electrospray device series suggested that their plume is dominated by monomer and dimer ions, with little if any evidence of charged droplets $^{13}$. Another possible reason for the five-stage energy distribution is the fragmentation effects occurring during the
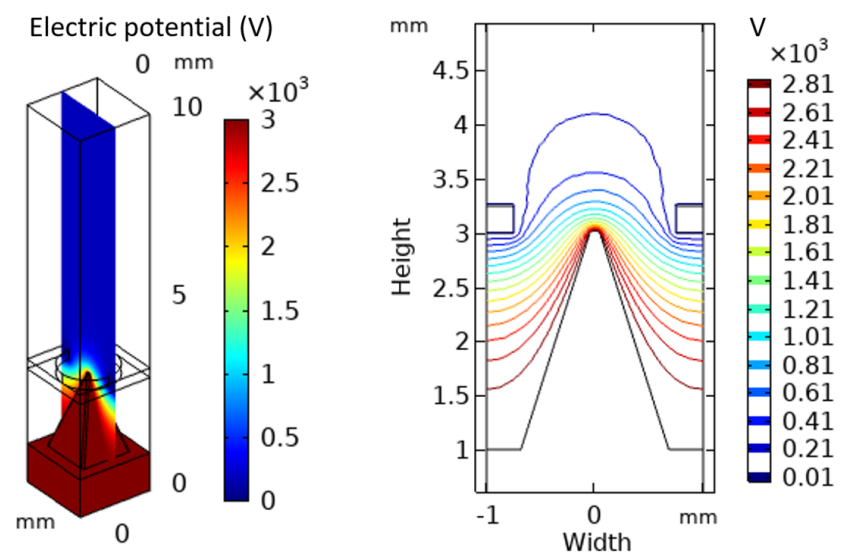

FIG. 12. Electric potential distribution near an electrospray emission tip.

emission of charged particles, which was observed in multiple previous electrospray source energy distribution experimental and simulation studies ${ }^{14-18,20,21}$.

In the PEILIS electrospray device, although the extractor is placed at the same plane as the emission tip, the acceleration electric field distorts significantly within a small region near the emitter tip, as illustrated in simulation results near an emission tip shown in Fig. 12. The computational domain has periodic boundary conditions, representing an infinite array of emission tips that takes electric field interference from adjacent tips into consideration.

Miller has found that the mean lifetime before EMI-BF 4 dimer structures fragmenting is around 1.49 to $1.60 \mu \mathrm{s}$ with an applied source voltage of $\pm 859 \mathrm{~V}^{16}$. In this paper, with the $35 \mathrm{~cm}$ distance between the electrospray extractor and RPA collector, the minimum acceleration voltage around $1800 \mathrm{~V}$, the flight time of dimer ions from the PEILIS to the RPA would take more than $10 \mu \mathrm{s}$, well exceeding the aforementioned dimer lifetime and resulting in a high likelihood of fragmentation. The fragmentation of the emitted ionic liquid molecules could occur within the intense electric field, or within the field-free region between the PEILIS electrospray device and the RPA, where the electric potential relative to the ground is less than $10 \mathrm{~V}$. This section studies the distribution of fragmentation locations and analyses the effects on the electrospray performance.

\section{A. Fragmentation within the Field-Free Region}

In an electrostatic acceleration system, a charged particle's kinetic energy variation is equal to the change of electrical potential energy,

$$
\begin{aligned}
& q_{1} U_{1}=\frac{1}{2} m_{1} v_{1}^{2}, \\
& q_{2} U_{2}=\frac{1}{2} m_{2} v_{2}^{2},
\end{aligned}
$$

where $m_{1}, v_{1}, q_{1}$ are the mass, velocity and charge of the ion before the fragmentation, respectively; $U_{1}$ is the acceleration 




FIG. 13. Illustration of fragmentation occurring after leaving the acceleration electric field.

voltage; $m_{2}, v_{2}, q_{2}$ are the mass, velocity and charge of the fragmented ion, respectively; and $U_{2}$ is the RPA voltage stopping the charged particle. For a dimer ion fragmenting into a monomer ion and a neutral molecule, the electrical charge of the ions does not vary during the fragmentation, $q_{1}=q_{2}$.

The process of the fragmentation occurring within the fieldfree region is illustrated in Fig. 13. Assuming the fragmentation occurs radially that is perpendicular to the flight direction, whilst the axial velocity does not change during the process, $v_{1}=v_{2}$, equation 4 can thus be rearranged to

$$
\frac{U_{1}}{U_{2}}=\frac{m_{1}}{m_{2}} .
$$

Equation 5 suggests that ratio of the initial acceleration voltage over the RPA stopping voltage is equal to the ratio of the charged particle mass before and after the fragmentation. Using EMI-BF 4 propellant, for the fragmentation of a dimer ion into a monomer ion and a neutral molecule, $m_{1} / m_{2}$ is approximately 2.78 for a positive ion, and approximately 3.28 for a negative dimer ion. If there were trimer ions, which, however, are deemed not present based on $\mathrm{ToF}$ characterisation results, fragmenting into dimer ions in the plume, the corresponding $U_{1} / U_{2}$ values are approximately 1.64 for positive ions and 1.70 for negative ions. In the case that a trimer ion fragments into a monomer ion with two neutral molecules, the $U_{1} / U_{2}$ values are approximately 4.56 for the positive and 5.56 for the negative.

In order to verify whether Stage 2 represents dimer ions fragmented after the extractor, the voltage data of the current intensity peak in Stage 2 is taken as $U_{2}$, and the applied emitter voltage is taken as $U_{1}$. Based on the RPA measurement data shown in Fig. 8, Fig. 9 and Fig. 10, the values of $U_{1} / U_{2}$ calculated using the Stage 2 and Stage 4 current intensity peak points at different voltages are summarized in Fig. 14. The $U_{1} / U_{2}$ values would vary depending on the points selected within the spread of current drops in Stage 2 and Stage 4, and based on this effect, the error ranges in Fig. 14 were calculated based on the starting and ending points of the spread of current drops. Although the calculated $U_{1} / U_{2}$ values from tested data are smaller than the theoretical values, they are relatively close and independent of the applied voltage. The results strongly suggest that the decreasing current in Stage 2 is a result of the fragmentation of dimer ions occurring in the field-free region downstream to the extractor. The slight

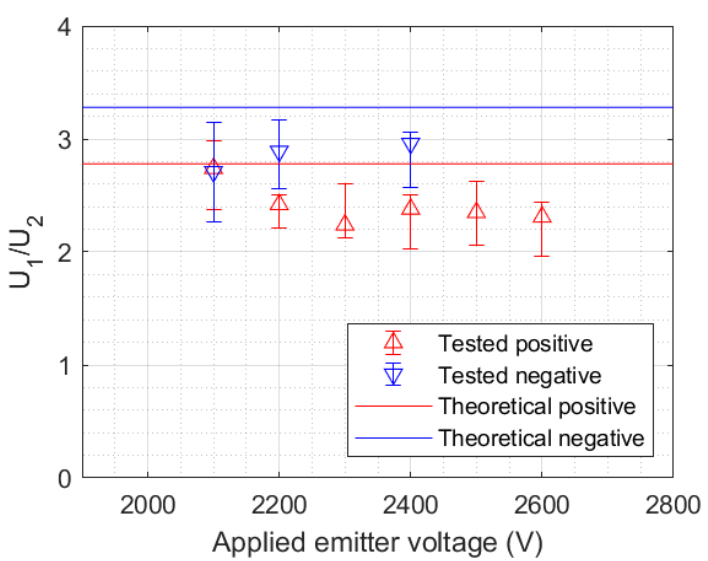

FIG. 14. Ratios of the applied emitter voltage over the voltage with the highest current intensity in Stage 2.

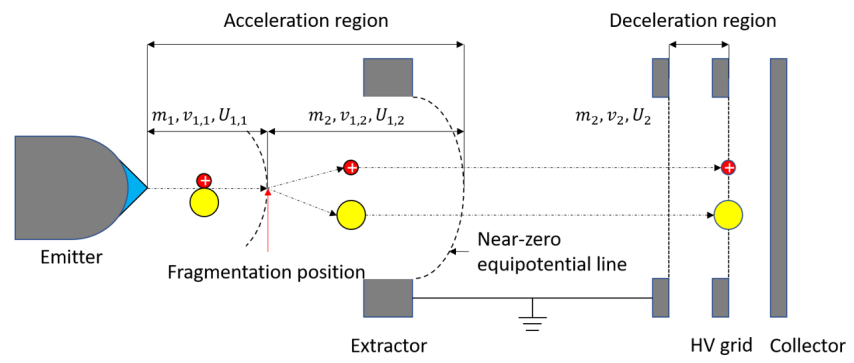

FIG. 15. Illustration of fragmentation occurring before leaving the acceleration electric field.

deviation values between the theoretical and measurement results could result from certain measurement errors, such as the milliseconds-range time delay from commanding the voltage output to the actual voltage output; or the data processing method, such as the selected data points within the decreasing current stages.

\section{B. Fragmentation inside the Acceleration Region}

If a proportion of dimer ions fragment before leaving the extractor, as illustrated in Fig. 15, the state of a particle can be divided into four stages: accelerating before fragmentation, accelerating after fragmentation, cruising after leaving the acceleration electric field, and decelerating as they enter the RPA electric deceleration field.

The energy and velocity equation during the first acceleration stage prior to fragmentation is

$$
q_{1} U_{1,1}=\frac{1}{2} m_{1} v_{1,1}^{2},
$$

whilst the equation for the second acceleration stage after fragmentation is

$$
q_{1} U_{1,2}=\frac{1}{2} m_{2} v_{1,2}^{2}-\frac{1}{2} m_{2} v_{1,1}^{2},
$$


and the equation for the deceleration stage is

$$
q_{1} U_{2}=\frac{1}{2} m_{2} v_{2}^{2},
$$

where $U_{1}=U_{1,1}+U_{1,2}$, the subscripts [1,1], [1,2] and [2] represent the states of the particle at the point of the fragmentation, the ending point of the acceleration region and the starting point of deceleration region, respectively. Both sides of equation 6 multiplied with $\frac{m_{2}}{m_{1}}$, the equation becomes

$$
\frac{q_{1} U_{1,1} m_{2}}{m_{1}}=\frac{1}{2} m_{2} v_{1,1}^{2} .
$$

By definition, $v_{2}=v_{1,2}$. Substituting the deceleration equation 8 and first acceleration stage equation 9 into the second acceleration stage equation 7 , the formula becomes

$$
q_{1} U_{1,2}=q_{1} U_{2}-\frac{q_{1} U_{1,1} m_{2}}{m_{1}},
$$

which can be simplified to

$$
U_{2}=U_{1,2}+U_{1,1} \frac{m_{2}}{m_{1}} .
$$

Defining the proportion of the acceleration voltage of the particle before fragmentation over the overall acceleration voltage, $r_{p}=U_{1,1} / U_{1}$, then

$$
\frac{U_{1}}{U_{2}}=\frac{1}{1+r_{p}\left(\frac{m_{1}}{m_{2}}-1\right)} .
$$

The relationship between $r_{p}$ and the ratio of full acceleration voltage versus the stopping voltage of the particle, $U_{1} / U_{2}$, is shown in Fig. 16. As $r_{p}$ approaches zero, the dimer ions would fragment immediately after being extracted and before gaining any acceleration; therefore, the fragmented monomer ions are accelerated and stopped by near full acceleration voltage, indicating Stage 4 in RPA current curves. As $r_{p}$ increases, a dimer ion would gain more energy before fragmenting into a monomer ion and a neutral molecule. The energy imparted to the neutral molecule would not be affected by the deceleration field, resulting in energy loss in the monomer ion charged particle with a lower RPA stopping voltage. As $r_{p}$ approaches 1, the dimer ions tend to fragment near the end of the electric acceleration field. The energy loss to the fragmented neutral particle would be higher and the RPA stopping voltage would be the lower, approaching Stage 2 of RPA current curves, where the fragmentation occurs after leaving the extractor. To summarise, the analysis suggests that the current decreasing Stage 3 is likely a result of fragmentation occurring before leaving the electric acceleration field.

Within Stage 3, the RPA current gradually decreases with the RPA-HV sweep potential: from the end of Stage $2\left(r_{p}=1\right)$, where fragmentation occurs within the field-free region, to the start of Stage $4\left(r_{p}=0\right)$, where there is no fragmentation. As a result, the gradual decrease in current throughout Stage 3 corresponds to the $r_{p}$ values ranging from 0 to 1 , suggesting that the fragmentation occurs throughout almost the entire electric acceleration field.

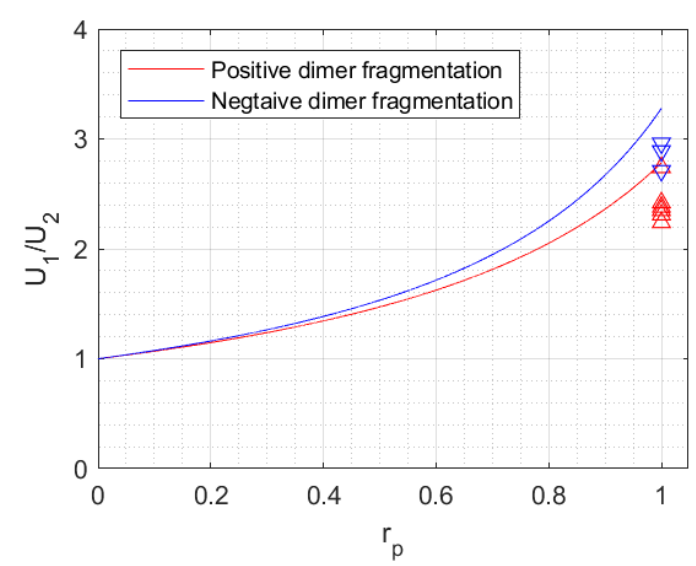

FIG. 16. The relations between the potential where particle fragmentation occurs and the relative stopping voltage applied to the RPA$\mathrm{HV}$ grid, in Stage 3. The scattered data points on the right end represent the calculated experimental $U_{1} / U_{2}$ values in Stage 2 from Fig. 14 , whose error ranges are removed to avoid confusion. The left end with the $U_{1} / U_{2}$ value of 1 represents Stage 4 .

Overall, the RPA current curves demonstrate a continuous energy loss trend, suggesting the dimer ions fragmentation occurs before and after leaving the acceleration field. The proportion of the dimer ions fragmenting at different locations in the acceleration field can be estimated based on Fig. 9. The proportion of the current drop in Stage 2 ranges from $45 \%$ to $55 \%$, deemed as the proportion of fragmented dimer ions after leaving the acceleration field. The current proportion of Stage 3, deemed a result of fragmentation within the acceleration field, is about $20 \%$ to $30 \%$. Stage 4 accounts for roughly $25 \%$ to $30 \%$ of total current drop, which can be unfragmented dimer ions, monomer ions or a mixture of both species.

Although PEILIS demonstrated high energy and high current emission consisting of dimer ions and monomer ions, emission of mono-energetic ions is usually the demanded operation type of an ion source. For further development of PEILIS electrospray ion source, it is recommended to reduce the proportion of fragmentation in the acceleration field, which possibly can be improved by reducing the size of the extractor aperture, therefore, the size of the acceleration field. Another suggestion is to achieve electrospray emission consisting solely of monomer ions with no presence of dimer ions to eliminate the energy variation from fragmentation, possibly through using other ionic liquids that have less propensity for dimer emission.

\section{CONCLUSIONS}

A retarding potential analyser was built and used to measure the energy distribution of the PEILIS electrospray source at different voltages from 2100 to $2600 \mathrm{~V}$ and from -2100 to $-2400 \mathrm{~V}$. Electric field simulation results illustrated a corrective factor between the applied RPA-HV grid potential and the actual spatial retarding potential, which was used to correct all 
the tested RPA data. With the retarding potential increases, the collected plume current decreases with five stages. The last current step stage represents the particles that have been fully accelerated, and the calculated voltage losses ranged from 298 $\mathrm{V}$ to $511 \mathrm{~V}$ with non-kinetic efficiency of $85.8 \%$ and $79.6 \%$ at applied emitter potentials of $-2100 \mathrm{~V}$ and $2600 \mathrm{~V}$, respectively. The high voltage losses are believed mainly a result of high resistance of the conductive ionic liquid path.

The two RPA stages with significant current steps represent the energy of particles with high current intensity, and the separation of these two current steps was believed a result of dimer ions fragmenting to monomer ions. The theoretical particle energy ratio before and after the fragmentation was calculated and found generally agreeing with the measurement results of the two significant current steps. About $45 \%$ to $55 \%$ of the emission current was deemed as fragmentation occurring after leaving the major acceleration electric field, and $20 \%$ to $30 \%$ of the emission current fragmented before leaving the acceleration field. As an initial investigation, the PEILIS electrospray ion source demonstrated high-energy and high-current-density emission of dimer and monomer ions, and further development is recommended to mitigate the detrimental effects from fragmentation on the ion source performance uniformity.

\section{DATA AVAILABILITY}

The data that support the findings of this study are available from the corresponding author upon reasonable request.

\section{ACKNOWLEDGEMENTS}

The work has been supported by the Royal Society Grant with the grant number RG150794, the UK Space Agency NSTP Pathfinder Grant under the grant number of NSTP3PF2-064, the Engineering and Physical Sciences Research Council under the grant number of EP/M508147/1, and the University of Southampton under the funding codes of F2ZEM4, F27K05, F26QTE and F22NS5.

\footnotetext{
${ }^{1}$ T. Nakashima, H. Wada, S. Morita, R. Erra-Balsells, K. Hiraoka, and H. Nonami, "Single-Cell Metabolite Pro fi ling of Stalk and Glandular Cells of Intact Trichomes with Internal Electrode Capillary Pressure Probe Electrospray Ionization Mass Spectrometry," Analytical Chemistry 88, 30493057 (2016).
}

${ }^{2}$ R. E. Huie, Z. B. Alfassi, B. L. Milman, and P. Neta, "Electrospray Ionization Mass Spectrometry of Ionic Liquids and Determination of Their Solubility in Water," Analytical and bioanalytical chemistry 377, 159-164 (2003).

${ }^{3}$ J. A. Loo, "Studying Noncovalent Protein Complexes by Electrospray Ionization Mass Spectrometry," Mass Spectrometry Reviews 16, 1-23 (1997).

${ }^{4}$ D. Li and Y. Xia, "Fabrication of Titania Nanofibers by Electrospinning," Nano Letters 3, 555-560 (2003).

${ }^{5}$ N. Bhardwaj and S. C. Kundu, "Electrospinning: A fascinating fiber fabrication technique," Biotechnology Advances 28, 325-347 (2010).

${ }^{6}$ S. Guilet, C. Perez-Martinez, P. Jegou, P. Lozano, and J. Gierak, "Ionic liquid ion sources for silicon reactive machining," Microelectronic Engineering 88, 1968-1971 (2011).

${ }^{7}$ C. Perez-Martinez, S. Guilet, J. Gierak, and P. Lozano, "Ionic liquid ion sources as a unique and versatile option in FIB applications," Microelectronic Engineering 88, 2088-2091 (2011).

${ }^{8}$ T. Xu, Z. Tao, and P. C. Lozano, "Etching of glass, silicon, and silicon dioxide using negative ionic liquid ion sources," Journal of Vacuum Science \& Technology B 36, 052601 (2018).

${ }^{9}$ S. Rauschenbach, F. L. Stadler, E. Lunedei, N. Malinowski, S. Koltsov, G. Costantini, and K. Kern, "Electrospray Ion Beam Deposition of Clusters and Biomolecules," Small 2, 540-547 (2006).

${ }^{10}$ V. N. Morozov and T. Y. Morozova, "Electrospray Deposition as a Method To Fabricate Functionally Active Protein Films," Analytical Chemistry 71, 1415-1420 (1999).

${ }^{11}$ D. Krejci, F. Mier-Hicks, R. Thomas, T. Haag, and P. Lozano, "Emission Characteristics of Passively Fed Electrospray Microthrusters with Propellant Reservoirs," Journal of Spacecraft and Rockets 54, 447-458 (2017).

${ }^{12}$ D. G. Courtney, N. Alvarez, and N. R. Demmons, "Electrospray Thrusters for Small Spacecraft Control: Pulsed and Steady State Operation," in 2018 Joint Propulsion Conference, AIAA Propulsion and Energy Forum (American Institute of Aeronautics and Astronautics, 2018) pp. 1-15.

${ }^{13}$ C. Ma, T. Bull, and C. N. Ryan, "Time-of-Flight Characterization of Electrospray Thrusters Using Porous Emitters with High Emission Currents," Submitted.

${ }^{14}$ M. R. Natisin and H. L. Zamora, "Performance of a Fully Conventionally Machined Liquid-Ion Electrospray Thruster Operated in PIR," in International Electric Propulsion Conference 2019, 19443 (2019) pp. 1-16.

${ }^{15} \mathrm{D}$. G. Courtney and H. Shea, "Fragmentation in Time-of-Flight Spectrometry-Based Calculations of Ionic Electrospray Thruster Performance," Journal of Propulsion and Power 31, 1500-1504 (2015).

${ }^{16}$ C. E. Miller and P. C. Lozano, "Measurement of the dissociation rates of ion clusters in ionic liquid ion sources," Applied Physics Letters 116, 3-8 (2020).

${ }^{17}$ D. G. Courtney, S. Dandavino, and H. Shea, "Comparing Direct and Indirect Thrust Measurements from Passively Fed Ionic Electrospray Thrusters," Journal of Propulsion and Power 32, 392-407 (2016).

${ }^{18}$ T. P. Fedkiw, Characterization of an Iodine-Based Ionic Liquid Ion Source and Studies on Ion Fragmentation, Ph.D. thesis, Massachusetts Institute of Technology (2010).

${ }^{19}$ B. L. P. Gassend, A Fully Microfabricated Two-Dimensional Electrospray Array with Applications to Space Propulsion, Ph.D. thesis, Massachusetts Institute of Technology (2007).

${ }^{20}$ P. C. Lozano, "Energy properties of an EMI-Im ionic liquid ion source," Journal of Physics D: Applied Physics 39, 126-134 (2005).

${ }^{21}$ T. Coles, T. Fedkiw, and P. Lozano, "Investigating Ion Fragmentation in Electrospray Thruster Beams," in 48th AIAA/ASME/SAE/ASEE Joint Propulsion Conference \&amp; Exhibit (2012). 